\title{
Oxaliplatin induced hyperexcitability syndrome in patient with appendiceal adenocarcinoma
}

\author{
Nicola Jabbour ${ }^{1^{*}}$, Maher Alchreiki ${ }^{2}$, Mohammad Hussain $^{3}$ \\ ${ }^{1}$ Hazard ARH Cancer Center, Hazard, USA; ${ }^{*}$ Corresponding Author: nicolajabbour@hotmail.com \\ ${ }^{2}$ Harlan ARH Hospital, Harlan, USA; alchreiki@yahoo.com \\ ${ }^{3}$ Johnson Medical Center, Smithfield, USA; doc.junaid@gmail.com
}

Received 25 April 2013; revised 26 May 2013; accepted 15 June 2013

Copyright (c) 2013 Nicola Jabbour et al. This is an open access article distributed under the Creative Commons Attribution License, which permits unrestricted use, distribution, and reproduction in any medium, provided the original work is properly cited.

\begin{abstract}
Oxaliplatin is a novel platinum compound with clinical activity against several solid tumors. It has been associated with sensory neuropathies and, rarely, a neuromyotonia-like hyperexcitability syndrome; a form of peripheral neuropathy manifested by tremor and twitching activity of muscles. We present a case of hyperexcitability syndrome developed during the treatment of stage III appendiceal adenocarcinoma with oxaliplatin-containing regimen and was successfully treated with Pregabalin.
\end{abstract}

Keywords: Oxaliplatin; Peripheral Neuropathy; Neuromyotonia; Hyperexcitability Syndrome; Appendiceal Adenocarcinoma; Sodium Channels

\section{INTRODUCTION}

Oxaliplatin, a platinum derivative, is an important component of the chemotherapy regimen used for both early and advanced stage colorectal malignancies. The main dose limiting toxicity of this agent is neurotoxicity. This adverse event has two distinct syndromes. Sensory neuropathy is correlated with long term administration of oxaliplatin [1]. It can also produce acute neurotoxicity, which usually develops shortly after infusion of oxaliplatin. Acute neurotoxicity may be triggered by exposure to cold, and is characterized with dysesthesia, parasthesia of the hands and feet, and pharyngo-laryngodysesthesia. [2,3], and infrequently, neuromyotonia-like hyperexcitability syndrome [4-6]. We present a case of neuromyotonia-like hyperexcitability syndrome that developed during adjuvant chemotherapy treatment for stage III appendiceal adenocarcinoma with oxaliplatin containing chemotherapy (oxaliplatin, leucovorin and 5 fluorouracil).

\section{CASE REPORT}

A 53-year-old white female presented at the emergency department with right lower quadrant abdominal pain and vomiting. A CT scan of the abdomen and pelvis revealed enlarged appendix along with inflammatory changes around the appendix and ileus. Subsequently, appendectomy was performed, and the pathology study was consistent with moderately differentiated adenocarcinoma of the proximal half of the appendix, with penetration of the visceral peritoneum, margins were not involved with invasive carcinoma. Upon this finding, the patient underwent right hemicolectomy, and the surgical pathology study showed metastatic adenocarcinoma in 2 out of 19 mesenteric lymph nodes. She had PET/CT scan which was negative, and the patient was staged at T4, N1, M0 (stage IIIB). Patient was started on adjuvant chemotherapy with Oxaliplatin, Leucovorin and 5 Fluorouracil. At completion of the second cycle, she experienced diffuse and significant muscle spasms involving her upper and lower extremities, along with peri-oral tingling and numbness, she required hospitalization , her neurological exam revealed increased tone of her upper and lower extremities bilaterally, serum electrolytes were within normal range including serum potassium and magnesium. The patient was started on Pregabalin at a dosage of 50 mg three times daily, and significant improvement of her symptoms occurred within 48 hours, patient was discharged on Pregabalin at the same dose. Adjuvant chemotherapy using same regimen was restarted as outpatient, throughout her adjuvant therapy, she was kept on Pregabalin, and she received one gram of magnesium sulfate and one gram of calcium gluconate before and after each Oxaliplatin infusion. The patient was able to complete her treatment. 


\section{DISCUSSION}

Oxaliplatin, a platinum derivative, is an antineoplastic agent indicated for both early and late stage colorectal cancers. Although it can cause multiple neurologic toxicities not limited to paresthesias, peripheral neuropathy and visual disturbances, special attention should be paid to a rare and clinically distinct feature known as neuromyotonia-like hyperexcitability syndrome, which warrants immediate management including, dose reduction or drug withdrawal depending on the severity of the symptoms. Clinically neuromyotonia is characterized by muscle stiffness, delayed muscle relaxation and twitching [7]. It may be autoimmune mediated, associated with neuropathy or a rare side effect of drugs, radiation therapy, or toxins $[8,9]$.

The patient discussed above developed this syndrome during her adjuvant chemotherapy with oxaliplatin, 5 fluorouracil and leucovorin for stage III appendiceal adenocarcinoma. A work up for autoimmune etiology yielded was negative and she had not taken any new medicines or herbal supplements.

While the exact mechanism of neuromyotonia in humans remains unknown, considerable emphasis is given to the persistent sodium channel activity or decreased potassium conductance as a cause of axonal hyperexcitability [10]. Other possible explanations include direct autoimmune blockade of voltage-gated potassium channels $[10,11]$ and exposure of sodium channels in paranodal regions suggesting that Oxaliplatin can act as a direct toxin against axonal ion channels.

Gabapentin in doses of 200 to $600 \mathrm{mg}$ daily has been used in the empirical treatment of neurotoxcity secondary to platinum drug. It also blocks the repetitive firing of neurons in vitro. Although it resulted in a partial or complete response in some patients, gabapentin failed to improve the acute neurosensory toxicity [12].

Pregabalin, an amino acid derivative of gamma-amino butyric acid has a similar pharmacological profile to gabapentin but has been shown in studies to provide equivalent efficacy to gabapentin at much lower doses given its higher bioavailability and pharmacokinetics, thus resulting in fewer dose-related adverse events. Its influences on the GABAergic neurotransmission, translates into analgesic and anxiolytic effects which led to its FDA approval for Diabetic neuropathy and post herpetic pain neuralgia [13].

We decided to treat our patient's Oxaliplatin induced Hyperexcitability with Pregabalin $50 \mathrm{mg}$ three times a day given its distinct advantages over gabapentin. She was able to finish her adjuvant chemotherapy course successfully without recurrence of this syndrome.

\section{CONCLUSION}

Oxaliplatin is an important component of the chemo- therapy regimens used for the treatment of both early and late stage colorectal malignancies; it can cause both acute and chronic neurologic toxicities. Hyperexcitability syndrome is a rare complication of oxaliplatin therapy. It is characterized by cramps, myotonia, and muscle twitching. It warrants immediate drug withdrawal or drug reduction.

\section{REFERENCES}

[1] Saif, M.W. and Reardon, J. (2005) Management of oxaliplatin-induced peripheral neuropath. Journal of Therapeutics and Clinical Risk Management, 1, 249-258.

[2] De Gramont, A., Figer, A., Seymour, M., et al. (2000) Leucovorin and fluorouracil with or without oxaliplatin as first-line treatment in advanced colorectal cancer. Journal of Clinical Oncology, 18, 2938-2947.

[3] Bleigberg, H. and De Gramont, A. (1998) Oxaliplatin plus 5-fluororuacil: Clinical experience in patients with advanced colorectal cancer. Seminars in Oncology, 25, 3239.

[4] Saif, M.W. and Hashmi, S. (2008) Successful amelioration of oxaliplatin-induced hyperexcitability syndrome with the antiepileptic pregabalin in a patient with pancreatic cancer. Cancer Chemotherapy and Pharmacology, 61, 349-354. doi:10.1007/s00280-007-0584-7

[5] Lahrmann, H., Albercht, G., Drlicek, M., Oberndorfer, S., Urbanits, S., Wanschitz, J., et al. (2001) Acquired neuromyotonia and peripheral neuropathy in a patient with Hodgkin's lymphoma disease. Muscle \& Nerve, 24, 834838. doi:10.1002/mus.1078

[6] Fort, F., Pretegiani, E., Battisti, C., Sicurelli, F. and Federico, A. (2009) Neuromyotonia as paraneoplastic manifestation of bladder canrcinoma. Journal of the Neurological Sciences, 280, 111-112.

doi:10.1016/j.jns.2009.01.022

[7] Leonard, G.D., Wright, M.A., Quinn, M.G., Fioravanti, S., Harold, N., Schuler, B., et al. (2005) Survey of oxaliplatin-associated neurotoxicity using an interview-based questionnaire in patients with metastatic colorectal cancer. BMC Cancer, 5, 116. doi:10.1186/1471-2407-5-116

[8] Auger, R.G. (1994) AAEM minimonograph: Diseases associated with excess motor unit activity. Muscle \& Nerve, 17, 1250-1263. doi:10.1002/mus.880171103

[9] Reeback, J., Benton, S., Swash, M. and Schwartz, M.S. (1979) Penicillamineinduced neuromyotonia. British Medical Journal, 1, 1464-1465. doi:10.1136/bmj.1.6176.1464

[10] Hart, I.K., Waters, C., Vincent, A., Newland, C., Beeson, D., Pongs, O., et al. (1997) Autoantibodies detected to expressed $\mathrm{K}+$ channels are implicated in neuromyotonia. Annals of Neurology, 41, 238-246.

[11] Nagado, T., Arimura, K., Sonoda, Y., Kurono, A., Horikiri, Y., Kameyama, A., et al. (1999) Potassium current suppression in patients with peripheral nerve hyperexcitability. Brain, 122, 2057-2066. doi:10.1093/brain/122.11.2057 
[12] Mitchell, P.L., Goldstein, D., Michael, M., Beale, P., Friedlander, M., Zalcberg, J., et al. (2006) Addition of gabapentin to a modified FOLFOX regimen does not reduce oxaliplatin-induced neurotoxicity. Clinical Colorectal Can-

\section{cer, 6, 146-151. doi:10.3816/CCC.2006.n.032}

[13] Frampton, J.E. and Foster, R.H. (2005) Pregabalin in the treatment of postherpetic neuralgia. Drugs, 65, 111-120. 